\title{
MEASUREMENT OF FLUID-STRUCTURE INTERACTION OF WIND TURBINES IN WIND TUNNEL EXPERIMENTS CONCEPT AND FIRST RESULTS
}

\author{
S. Nietiedt ${ }^{1, *}$, M. Goering ${ }^{1}$, T. Willemsen ${ }^{2}$, T. T. B.Wester ${ }^{3}$, L. Kröger ${ }^{3}$, G.Guelker $^{3}$, T. Luhmann ${ }^{1}$ \\ ${ }^{1}$ Jade University of Applied Sciences, Institute of Applied Photogrammetry and Geoinformatics (IAPG), Ofener Str. 16/19, 26121 \\ Oldenburg, Germany - (Simon.Nietiedt, Martina.Goering, Thomas.Luhmann)@jade-hs.de \\ ${ }^{2}$ University of Applied Sciences Neubrandenburg, Department of Landscape Sciences and Geomatics, Brodaer Str. 2, 17033 \\ Neubrandenburg, Germany - willemsen@hs-nb.de \\ ${ }^{3}$ University of Oldenburg, ForWind and Institute of Physics, Küpkersweg 70, 26129 Oldenburg, Germany - (tom.wester, \\ lars.kroeger, gerd.guelker)@uol.de
}

\section{Commission II}

KEY WORDS: Particle Image Velocimetry, Photogrammetry, Dynamic application, Wind tunnel, Fluid structure interaction, HighSpeed, Active grid

\begin{abstract}
:
Fluid-structure interactions are crucial for the design of rotor blades of wind power systems. Up to now, the mutual interactions between rotor blades and turbulent wind flows have been treated by complex simulations or were observed at individual discrete points. In this paper, a measurement concept is presented where spatial information of the motion/deformation of a rotating wind turbine as well as the wind flow are recorded in wind tunnel experiments. Wind flow and motion behaviour are recorded simultaneously and contactless. Techniques from the field of photogrammetry and flow measurement techniques are combined, resulting in high demands on the measurement concept. Furthermore, solutions for the realisation of a common coordinate system as well as for the synchronisation of both measuring systems are presented. In addition, the validation of the entire measurement concept is carried out based on of some wind tunnel tests in which a single rotor blade is used for the moment. This showed that the measurement concept and the proposed solutions for the simultaneous recording of wind flows and rotor blade movements are suitable in principle and that movements can be recorded and reconstructed with high accuracy.
\end{abstract}

\section{INTRODUCTION}

Wind turbines are becoming increasingly important for power generation. However, most wind turbines do not achieve the expected service life, as the turbulent atmosphere is a burden on the turbines in addition to their own weight and rotation. The loads are caused by the turbulent atmosphere and are referred to as fluid-structure interactions (FSI). These are the mutual reactions of the wind flow and circulating object (rotor blade). Additional vibrations of a structure caused by the wind flow change the wind flow again. Although FSI are of great importance for the design of rotor blades, they have so far been largely investigated by complex simulations. The aim of the project "TurbuMetric" is to develop a measurement concept with which the interactions of rotating wind turbines and turbulent wind flows can be investigated directly in wind tunnel experiments.

This paper presents a concept that combines different optical measurement techniques in order to simultaneously record wind flows and blade deformations of miniaturised wind turbines in wind tunnel experiments.

In addition to the introduction, the paper is divided into three sections. Chapter 2 presents the previous approaches of photogrammetry that can be used to investigate wind turbines. In addition, the components of flow measurement techniques as well as previous experimental investigation approaches of FSI are explained. In chapter 3, the developed measurement concept is presented and various challenges are explained in more detail. Chapter 4 summarizes the results of the first experiments, followed by a discussion of the results in chapter 5 .

\section{RELATED WORK}

\subsection{Photogrammetry measurements on wind turbines}

In the field of wind turbine research, techniques from the field of photogrammetry have so far mainly been used for the recording of deformations in open field tests. Winstroth et al. (2014) describe a high-speed stereoscopic measurement system that can record the deformations of real wind turbines during operation with the aid of an additional texture attached to the rotor blades. The disadvantage of this method is the fact that a texture is applied which leads to long downtimes. As an alternative approach, Göring and Luhmann (2017) describe a procedure in which the additional texture can be dispensed with. Four 1D laser units are used which, in combination with a camera, determine the torsion and movement of rotor blades. In addition, it is possible to determine the deformations of operating systems photogrammetrically using a silhouette-based approach (Jepping and Luhmann, 2016). Only the silhouette of the rotor blades and previous knowledge of the shape of the rotor blades and the deformation behaviour is required for this method. The deformations can be determined using an extended adjustment approach integrating an elastic beam model,

\footnotetext{
* Corresponding author
} 
whereby the quality is strongly dependent on the quality of the previous knowledge, among other factors. However, all these approaches cannot be applied to wind tunnel tests without further effort, since the test environment, the object size and the simultaneous FSI observation create a completely different situation.

\subsection{Wind flow measurement techniques}

A number of different methods can be used to record wind flows. Procedures from the field of anemometry can obtain only punctual, but temporal high-resolved information about the flow velocity. Optical measurement techniques provide the area-wide acquisition of information on the flow behaviour of a fluid. Techniques from the field of Particle Image Velocimetry (PIV) have established themselves as standard methods which have been used successfully for more than 25 years in the field of wind turbine investigation (Whale and Anderson, 1993).

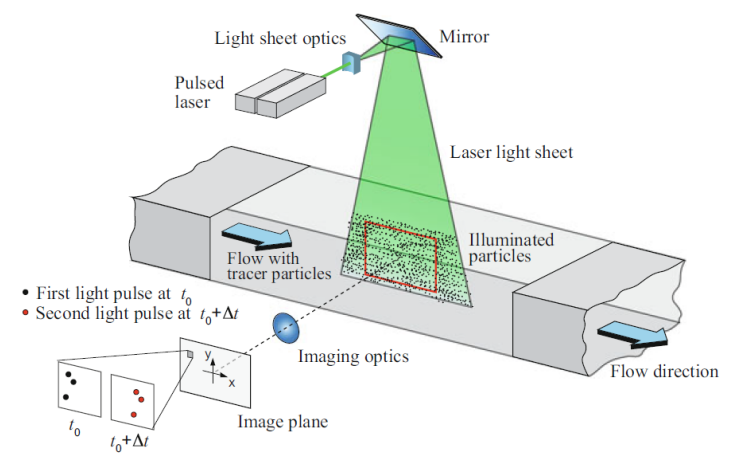

Figure 1. Exemplary representation of PIV (Raffel et al., 2018, p.8)

In PIV methods, the fluid to be investigated is mixed with small particles (tracers) which follow the flow without slip. As shown in Figure 1, these are illuminated by a laser plane and observed with at least one high-speed camera. By comparing two consecutive particle images with known recording times, two velocity components can be determined. If more than one camera are used, it is also possible to determine the third flow component. Due to the sometimes small particle size $(\approx 1 \mu \mathrm{m})$ and the high particle density, blurred or noisy images lead to a reduced data quality. Therefore, high demands are placed on the experimental setup. Scheimpflug adapters and sharp-edged band pass filters are used which are only permeable to the laser light used. However, only flow parameters within the spanned laser plane can be determined. A further development is the TomoPIV, with which volume-related information can be determined. Detailed descriptions of the various extensions are described in Raffel et al. (2018).

\subsection{Measurement techniques for fluid-structure interaction}

Wind flows can be well investigated using the techniques described above. Currently, there are only a few possibilities for the investigation of FSI in the field of wind energy research. Tian et al. (2014) describes wind tunnel experiments in which the reaction of a rotating miniaturised wind turbine is measured using a force sensor and the changed wind flow is recorded using a PIV system. Measurement relevant questions (synchronisation of the sensors with the rotating turbine and the turbulent wind flow or verification of the measurement data) are not dealt with in detail. In addition, this approach does not provide any information about the deformations that occur, which is of particular interest in the case of torsion of the rotor blades. Approaches which collect information about deformations and wind currents are known from biological (Nila et al., 2016) and industrial applications (Weichselbaum et al., 2016 u. Acher et al., 2019). Relevant information about the object reactions is derived from the results of photogrammetric stereo images and combined with the results of the respective PIV system. Thus, partly three-dimensional deformation and flow information is available. The required synchronisation takes place by means of an external trigger signal. However, these applications differ from those of a rotating wind turbine in terms of dynamics and object size.

\section{CONCEPT AND REALISATION}

The concept is based on the use of a three-bladed wind turbine model described in detail in Berger et al. (2018). The scaling factor of the rotor blade length is $1: 70$, so the rotor blades made are $90 \mathrm{~cm}$ long. The rotor blades of the model rotate at the tip with up to $48 \mathrm{~m} / \mathrm{s}$, which corresponds to a scale of 50:1 to a real turbine. Deformations in the range of $3 \mathrm{~cm}$ and vibrations of up to $30 \mathrm{~Hz}$ are expected. The turbulent wind flow in the turbulence wind tunnel of the University of Oldenburg is based on real wind conditions. The aim of the measurement concept is now to record the occurring FSI simultaneously and area-wide and to represent them in a common coordinate system.

\subsection{General concept}

Figure 2 shows the conceptual experimental setup. The turbulent wind flow is generated by a special three-dimensional active grid mounted in front of the outlet of the wind tunnel (3 $\mathrm{m} \times 3 \mathrm{~m}$ ) described in Kroeger et al. (2018). The turbulent wind flows (blue arrows) circulate around the model wind turbine and are observed by the flow measurement technique. For this purpose, a stereo PIV system is used whose cameras are shown in orange and arranged at right angles to the wind flow. The required laser area is stationary for the whole experiment and is generated by a powerful laser (green). The other four highspeed cameras (blue) together form a photogrammetric measurement system that observes the targeted rotor blades. In addition, tower movements can be recorded which contribute to a further understanding of FSI. Due to the high rotational speeds of $45 \mathrm{~m} / \mathrm{s}$, a short exposure time is required. For this purpose, three LED spotlights (yellow) are used, which illuminate the entire measuring volume as homogeneous as possible.

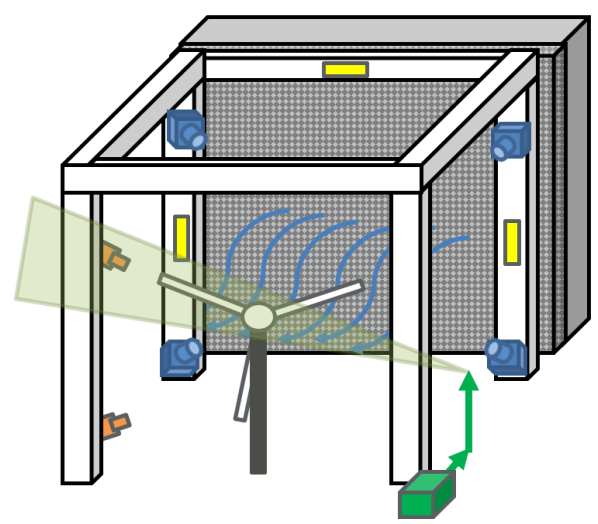

Figure 2. Schematic diagram of measurement concept

The realisation of the concept requires several steps. First, the two measurement systems are calibrated independently of each other. For the PIV system, a multi-level target (red marked test 
field in Figure 3) is used to determine the parameters of the interior and exterior orientation on the basis of space resections. The determination of the interior and exterior orientations of the cameras of the photogrammetric measurement system is performed by a test field calibration using a modified bundle adjustment approach. The modified mathematical model corresponds to the configuration that the relative position and orientation of the four cameras to each other is constant. Therefore, only for the images of one camera (reference camera) the exterior orientations within the bundle adjustment are determined. The exterior orientations of the remaining images can be calculated using the relative orientations of the remaining cameras to the reference camera determined in the adjustment (Luhmann et al, 2019, p. 362). It is assumed that the orientations of both measurement systems do not change during the experiment, so that the predetermined relative orientation of the two measurement systems to each other is also constant. During the experiment, the images from both systems are recorded and then processed in post-processing. The determined flow components are thus in the coordinate system of the multilevel target and the discrete observed measuring marks or the reconstructed rotor blade surface are given in the coordinate system of the photogrammetric measurement system. The calculation of the reconstructed surface or the coordinates of the discrete measuring marks as well as the determination of the flow vectors takes place in each epoch. Then the flow information and $3 \mathrm{D}$ coordinates of the respective rotor blade can be transformed into a uniform coordinate system.

\subsection{Challenges}

3.2.1 Coordinate system: Each measurement system has its own coordinate system. A transformation into a uniform coordinate system is necessary for the analysis of FSI. However, the measurement fields of both systems are different. While the photogrammetric measurement system can observe an area of $2 \mathrm{~m} \times 2 \mathrm{~m}$, the measurement field of the PIV system is only $22 \mathrm{~cm} \times 47 \mathrm{~cm}$. Therefore, the distribution of the homologous points required for transformation is limited to the small PIV area. Small deviations in the control point pairs can thus produce higher deviations in the remaining photogrammetric measurement field.

For the combination of both systems a six parameter transformation is used. It describes a transformation from the coordinate system of the multi-level target to the coordinate system of the photogrammetric measurement system. The required transformation parameters are determined using a special test field (combi target) shown in Figure 3.

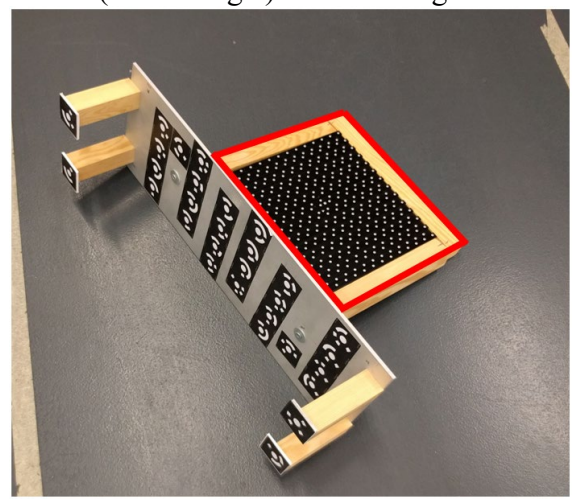

Figure 3. Combi target
The combi target consists of photogrammetric marks and the multi-level target (red), which is used for the calibration of the PIV system. The design allows both measurement methods to simultaneously acquire the test field and evaluate the corresponding measurement marks. The combi test field is calibrated in advance so that all measurement marks are present in the coordinate system of the multi-level target. The photogrammetric measurement marks are measured during the calibration process of the PIV system so that the required transformation parameters can be determined via the homologous points. All information is now available in one common coordinate system.

3.2.2 Synchronisation: Exact synchronisation between the two measurement systems is essential so that both information about the FSI are available in the same time system and a combined analysis is possible. Even small asynchronicities between the cameras within a measurement system can lead to systematic shifts and increased standard deviations of the determined object coordinates (Raguse, 2007).

Trigger boxes that send a TTL signal to the cameras are used for synchronisation within the measurement systems. The synchronisation of all six cameras with a trigger box is not possible due to hardware-specific reasons. Therefore, the synchronisation is solved using the active grid, which sends a start/stop signal as a square wave signal to the two trigger boxes. The respective recording is thus carried out independently of each other, enabling the use of different acquisition frequencies. In addition, the amount of data can be reduced, since the recording duration can be adjusted via the active grid. However, it can lead to asynchronicity between the photogrammetric and flow measurement system due to the different signal processing within the trigger boxes and cable lengths.

3.2.3 Influence of the test environment: A further challenge in the acquisition of FSI is the simultaneous use of the two measurement systems. For the PIV system, tracers are added to the wind flow so that a fog can be generated which may have a negative effect on the photogrammetric measurement system. Furthermore, the cameras of the photogrammetric system can be overdriven and the sensors destroyed if they are hit by highenergy laser light. The photogrammetric measurement system can also have a negative effect on the PIV measurement, since the LED illumination used illuminates the particles as well. This can lead to particle shadowing or increased image noise, which makes the evaluation of the particle images more difficult.

Experimental investigations have shown that the additional LED lighting has no influence on the PIV system. This is due to the sharp-edged band pass filters of the PIV cameras which allow the wavelength of the laser to pass through. Although the LED spotlights also cover the transparent wavelength range $(530-534 \mathrm{~nm})$, the intensity is significantly lower than that of the laser used. Since the laser is usually not aligned in the direction of the camera, only reflections can cause the camera sensors to overdrive. However, this can be prevented by taping off the corresponding reflection points.

\subsection{Parametrisation and accuracy estimation}

The selection of the recording configuration depends on the measurement environment and the available equipment. The situation described above requires a measuring field of $2 \times 2$ meters. Therefore, the photogrammetric measuring system is installed with a recording distance of 3 meters to the miniaturised wind turbine. The distance between the individual 
cameras is 3 meters. This ensures that the cameras do not stand within the turbulent wind flow which might impact the particle stream. If the cameras are used with lenses with a focal length of $21 \mathrm{~mm}$, this results in a GSD of $1.57 \mathrm{~mm}$. The optimum exposure time results from the rotational speed of the rotor blades. This is fixed at $10 \mu \mathrm{s}$, so that the motion blur at the tip of the rotor blades reaches 0.3 pixels. The recording frequency is set as a function of the expected vibrations and overlays and results to $500 \mathrm{~Hz}$.

For a theoretical accuracy estimation of the photogrammetric measurement system, a Monte Carlo simulation (MCS) is used (Hastedt, 2004). A spatial intersection is performed 10000 times. In each individual calculation, the parameters of the interior and exterior orientation as well as the image measurement accuracy of all four cameras are altered within a specific noise level. These changes correspond to real/expected standard deviations as shown in Table 1. The simulation shows that a $3 \mathrm{D}$ point accuracy of $0.4 \mathrm{~mm}$ is realistic. In addition, the MCS shows that the accuracy is most sensitive to uncertainties of convergence angles and base lengths. However, possible effects of dynamic recording or systematic movements between the four cameras are not considered, so the desired accuracy is set to $0.5 \mathrm{~mm}$.

\begin{tabular}{|c|c|}
\hline Parameters & Standard deviation \\
\hline Focal length $(\mathrm{mm})$ & 0.0009 \\
Principal point $\mathrm{x}^{ } \mathrm{c}(\mathrm{mm})$ & 0.0012 \\
Principal point $\mathrm{y}^{ }{ }_{0}(\mathrm{~mm})$ & 0.0008 \\
A1 & $5.17251 \mathrm{E}-07$ \\
A2 & $7.90947 \mathrm{E}-09$ \\
A3 & $3.5976 \mathrm{E}-11$ \\
B1 & $6.89912 \mathrm{E}-07$ \\
B2 & $6.01691 \mathrm{E}-07$ \\
C1 & $7.65327 \mathrm{E}-06$ \\
C2 & $8.05538 \mathrm{E}-06$ \\
Image measurement accuracy (Pixel) & 0.1 \\
\hline
\end{tabular}

Table 1. Standard deviations for the Monte Carlo simulation

In order to estimate the influence of asynchronicity within the photogrammetric measurement system, a simulation is performed. The parameters of the interior and exterior orientation of the camera system as well as target coordinates of a rotating wind turbine are required. The image coordinates can be calculated from the target coordinates, whereby the cameras are based on different times and thus different object coordinates. With the calculation of a spatial intersection, new object coordinates are calculated whose deviations from the target coordinates depend only on the simulated asynchronicity. This allows to estimate the influence of asynchrony on the measurement accuracy. Figure 4 shows the resulting deviations from the target coordinates of a point at the tip of the rotor blade as an example for a camera of the setup. In order to detect position-dependent effects, the occurring deviations are shown at four different positions rotated by $90^{\circ}$.

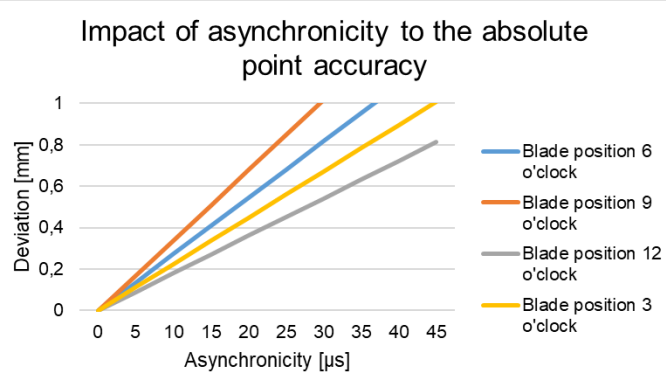

Figure 4. Exemplary representation of the effects of asynchronicity to point accuracy
It can be seen that there is a linear progression for all positions and that the highest deviations occur at 9 o'clock position. As a consequence, the highest sensitivity to asynchronicity occurs at this position. With regard to the desired accuracy level of 0.5 $\mathrm{mm}$, a maximum asynchronicity of $12.5 \mu \mathrm{s}$ is permissible.

\section{EXPERIMENTS}

\subsection{Construction and environment}

For the validation of the measurement concept, experiments were carried out in a wind tunnel of the University of Oldenburg. The wind tunnel according to Göttingen design has an outlet of $0.8 \mathrm{~m} \mathrm{x} 1 \mathrm{~m}$, so that it differs clearly from the larger wind tunnel. Consequently, no rotating wind turbine is used, but a mounted rotor blade of the model wind turbine which reduces the complexity of the experiment. Therefore, the realisation of the measurement concept and the data fusion as well as the verification of the photogrammetric measurement system are at the focus of the experiments.

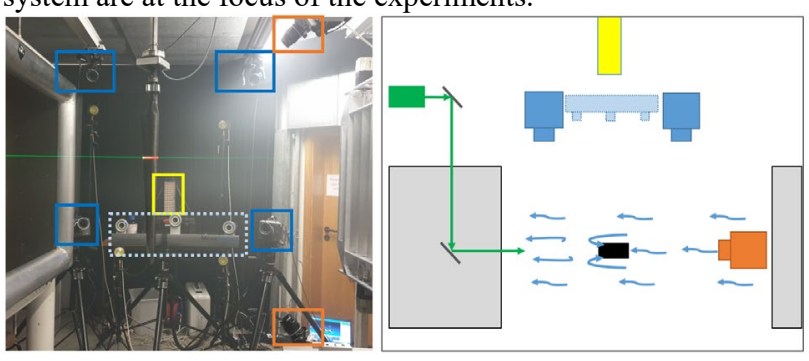

Figure 5. Left: Measurement setup in the wind tunnel.

Right: Schematic top view of the experimental setup

Figure 5 shows the test setup in the wind tunnel which corresponds to an approximation of the measurement setup already described. The two cameras of the PIV system (orange) are aligned in wind direction. The laser is positioned outside the experiment, which is why the laser beam illuminates the tracers and the rotor blade via mirrors. The laser plane is located approximately $25 \mathrm{~cm}$ below the blade root (see red stripe in Figure 6). The illumination for the photogrammetric measurement system (blue) consists of an LED spotlight (yellow) mounted behind the measurement system. The experimental setup was extended by an additional photogrammetric measurement system (light blue), namely the AICON MoveInspect HF which is used for the verification of the 4-camera system. It has an absolute measuring accuracy of $0.15 \mathrm{~mm}$ in a distance of $1.5 \mathrm{~m}$ at a recording frequency of up to 500 Hertz in full screen mode (AICON, 2013).

\subsection{Parametrisation and workflow}

In the wind tunnel, a 2D active grid (Wester 2018) is used to modify the wind flow periodically to induce loads by changing angle of attacks. During the experiment the flow was modified with a frequency of $8 \mathrm{~Hz}$. Thus it is to be expected that the main oscillations of the rotor blade also amount to $8 \mathrm{~Hz}$. The wind speed is constant for all experiments and amounts to approx. 15 $\mathrm{m} / \mathrm{s}$. The test duration is 30 seconds.

Two Phantom Miro 320S cameras with $50 \mathrm{~mm}$ lenses and Scheimpflug adapters are used for the PIV system. The cameras have a distance of about $1.5 \mathrm{~m}$ to the rotor blade and acquire the wind flow with a recording frequency of $1000 \mathrm{~Hz}$. The required particles consist of Di-Ethyl-Hexyl-Sebacate (DEHS) and are introduced into the wind tunnel using a seeding generator.

The photogrammetric measurement system consists of two PCO Dimax HD + and two PCO CS3 cameras each, which are used 
with $21 \mathrm{~mm}$ lenses. The base lengths and distances are about $1.5 \mathrm{~m}$. This results in a very good geometrical triangulation angle. A Monte Carlo simulation carried out in advance shows that $3 \mathrm{D}$ accuracies of $0.3 \mathrm{~mm}$ can be expected if discrete points are observed. The recording frequency and exposure time are chosen with regard to the expected oscillations and are $100 \mathrm{~Hz}$ or $80 \mu \mathrm{s}$, respectively. However, the rotor blade cannot be observed for the entire duration of the test because the data storage capacities are restricted. Therefore, the acquisition time is limited to five seconds. It can be assumed that the movements of the rotor blade remain constant throughout the entire duration of the test.

The coordinate system in which both the movements and the velocity vectors of the rotor blade are to be present is realised by the discrete measurement marks of the photogrammetric measurement system shown in Figure 6. The origin of the coordinate system is defined by a measuring mark (white point). The $\mathrm{X}$-axis (red) is directed in wind direction and the $\mathrm{Y}$-axis to the blade tip of the rotor blade. The Z-axis completes the righthanded coordinate system. The angle of attack of the rotor blade relative to the incoming flow is $15^{\circ}$.

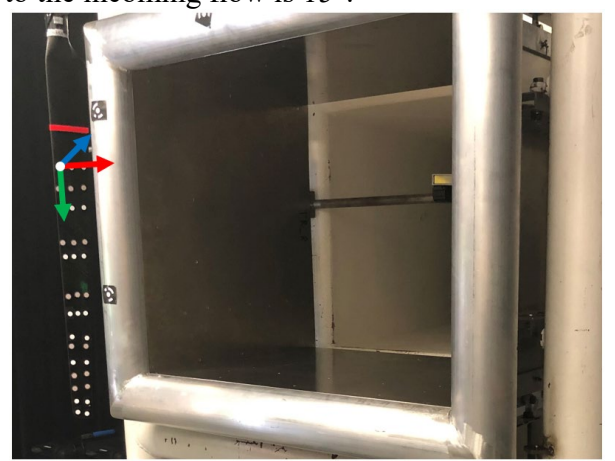

Figure 6. Mounted rotor blade in the wind tunnel; Realisation of the reference coordinate system

The experiments are carried out according to the conceptual procedure described above. In the first step, the two measurement systems are calibrated. When calibrating the PIV system, the presented combi test field is used and simultaneously captured by the photogrammetric system. This ensures that the generated data can then be transferred to a common coordinate system. The required synchronisation is performed by the respective trigger boxes and the active grid. The active grid sends a trigger signal to the trigger boxes of the two camera systems, which in turn synchronise the respective cameras. After calibration, the FSIs are observed and the recorded images are evaluated in post-processing. For the evaluation of the PIV data the commercial software $\mathrm{DaV}$ is from LaVision $\mathrm{GmbH}$ is used (LaVision, 2019). For the evaluation of the photogrammetric measurement system the software MoveInspect from AICON 3D Systems GmbH is applied. The following transformation of the results into the final system is done with AICON 3D Studio.

\subsection{Results}

All five experiments carried out in the wind tunnel lead to similar results, so that the following results are valid for all experiments.

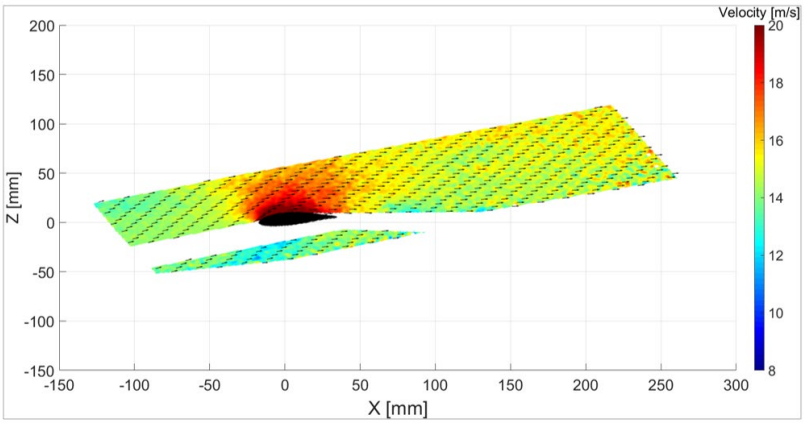

Figure 7. Instantaneous flow field around model wind turbine blade in reference coordinate system

Figure 7 shows the determined flow field and a schematic representation of the rotor blade (black). The wind speeds are shown in colour and are given between 10.5 and $23.5 \mathrm{~m} / \mathrm{s}$. The wind directions are represented by the arrows. The empty areas around the rotor blade caused by the shading of the rotor blade are conspicuous, but to be expected.

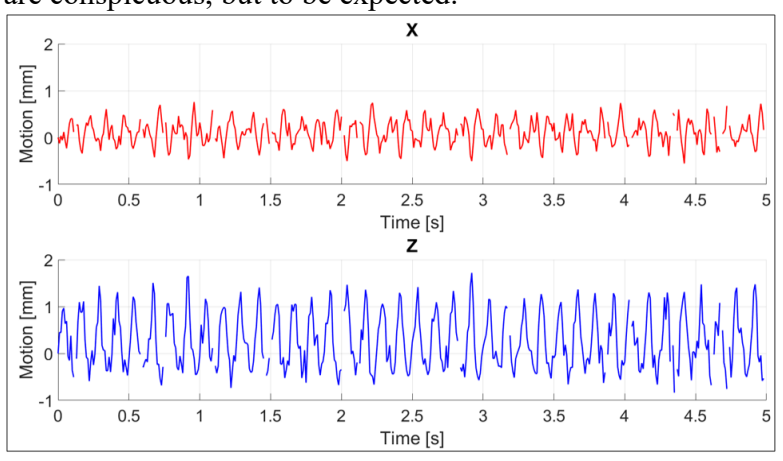

Figure 8. Motion of the rotor blade in $\mathrm{X}$ und $\mathrm{Z}$ direction

Figure 8 shows the movements of a point in the middle of the rotor blade in X- and Z-direction, which have been adjusted for the first epoch. The movements in the Y-direction are not shown, as they correspond to the previously determined measurement noise of $0.04 \mathrm{~mm}$. The movements follow a sinusoidal course and average $0.11 \mathrm{~mm}$ in X-direction and 0.27 $\mathrm{mm}$ in Z-direction. The largest movements can be seen in the recording direction of the cameras (Z-direction), which are up to $1.72 \mathrm{~mm}$. A closer analysis of the occurring frequencies of the three-dimensional movements is done by means of the amplitude spectrum, which can be seen in Figure 9.

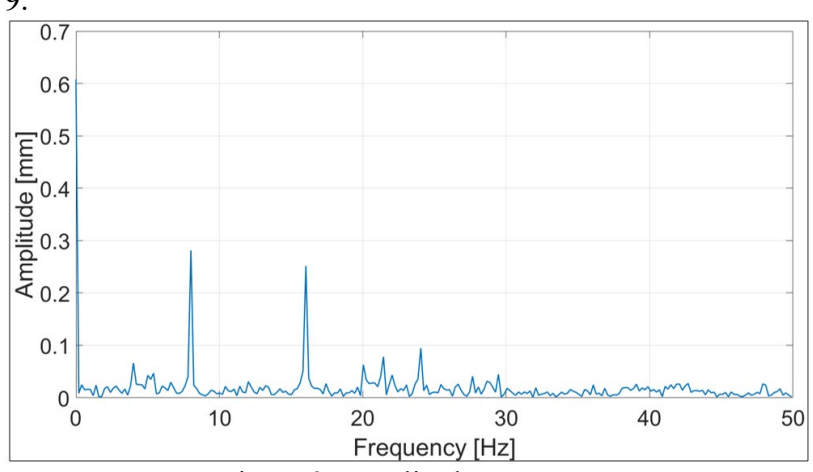

Figure 9. Amplitude spectrum

This can be used to demonstrate that the frequencies $8 \mathrm{~Hz}$ and $16 \mathrm{~Hz}$ are significantly present in the data set. This confirms the assumption that the frequencies of the turbulent wind flow become visible in the movements of the rotor blade. 
The data of the 4-camera system can be compared with the data of the MoveInspect system. Verification is not possible because the MoveInspect measurement accuracy of $0.15 \mathrm{~mm}$ is insignificantly higher than the theoretically estimated accuracy of the 4-camera system. Therefore, only a coordinate comparison is carried out that does not reveal any noticeable deviations. The RMS value of the three-dimensional motion deviation with respect to MoveInspect is $0.41 \mathrm{~mm}$. It is therefore assumed that the desired accuracy of $0.5 \mathrm{~mm}$ is achieved with this setup.

For the analysis of fluid-structure interactions the combined representation of flow and motion information in a coordinate system is aimed at. A validation of the solution strategy described above is difficult. Therefore, a visual control of the flow data, which were recalculated into the images of the photogrammetric measurement system, was carried out with the position of the red adhesive tape (Figures 5 and 6). The comparison shows an offset of $0.8 \mathrm{~mm}$ in object space, which corresponds to 1 pixel in image space and which is accurate enough for the analysis of fluid structure interactions.

\section{SUMMARY AND OUTLOOK}

This paper presents a measurement concept for contactless and full coverage detection of fluid-structure interactions on rotating wind turbines in wind tunnel experiments. For this purpose, photogrammetric methods are combined with a PIV system to provide three-dimensional information about the wind flow and the movements of the wind turbine in a uniform time and coordinate system.

A first validation of the concept took place in the medium sized wind tunnel of the University of Oldenburg. Due to the small size of the wind tunnel outlet, a mounted rotor blade is used instead of a rotating system. This test has demonstrated that, in principle, the presented concept is suitable for the acquisition of FSI. Although an additional photogrammetric measurement system of higher accuracy is used, only coordinate comparisons could be used for accuracy estimation. An intensive accuracy investigation regarding the systematic and random influences in the measurement concept has not yet been carried out.

Future developments are aimed at validating the accuracy of the measurement concept with superior accuracy and testing it on a rotating wind turbine within the large turbulent wind tunnel. In addition, the photogrammetric measurement system will be optimised in such a way that high-frequency movements of the whole rotor blade are reconstructed with high accuracy. For this purpose, methods such as OSGM (Bethmann and Luhmann, 2017) or silhouette-based approaches (Jepping and Luhmann, 2016) will be investigated and further developed in order to be able to dispense with the necessity for additional targeting.

\section{ACKNOWLEDGEMENTS}

This work is based on the project "TurbuMetric". Funded by the European Regional Development Fund (EFRE, ZW 685017185).

\section{REFERENCES}

Acher, G. Thomas, L. Tremblais, B. Gomit, G. Chatellier, L. David, L. 2019: Simultaneous measurements of flow velocity using TOMO-PIV and deformation of a flexible wing. $13^{\text {th }}$ international symposium on particle image velocimetry - ISPIV 2019, Munich, 619-628.

AICON 3D Systems. 2015. MoveInspect XR8 | HF4 technical specification. Data sheet.

Berger, F., Kröger, L., Onnen, D., Petrović., Kühn, M. 2018: Scaled wind turbine setup in a turbulent wind tunnel. Journal of Physics: Conference Series 1104 (2018) 012026.

Bethmann, F. and Luhmann, T. 2017.Object-based semi-global multi image matching. Journal of Photogrammetry, Remote Sensing and Geoinformation Science, 85, 349-364.

Hastedt, H. 2004: Monte-Carlo-Simulation in close-range photogrammetry. The international archives of the photogrammetry, remote sensing and spatial information sciences, 35, 18 - 23.

Göring, M. and Luhmann, T. (2017): Entwicklung eines fächerartigen Distanzmesssystems zur Messung von Rotorblättern - Konzept, Orientierung und erste Ergebnisse. In Luhmann/Schumacher (eds.): Photogrammetrie, Laserscanning, Optische 3D-Messtechnik - Beiträge der 16. Oldenburger 3DTage, Wichmann Verlag, Offenbach/Berlin, pp. 52-65

Jepping, C. and Luhmann, T. 2016. Object deformations from image silhouettes using a kinematic finite element beam model. The international archives of the photogrammetry, remote sensing and spatial information sciences. XLI-B5, 41 - 47.

Kröger, L., Frederik, J., van Wingerden, J.-W., Peinke, J., Hölling, M. 2018: Generation of user defined turbulent inflow conditions by an active grid for validation experiments. Journal of Physics: Conference Series, 1037, 052002

LaVision GmbH, 2019: DaVis 10. Product Information. www.lavision.de/en/products/davis-software (29 October 2019).

Luhmann, T., Robson, S., Kyle, S., Boehm, J. (2019): CloseRange Photogrammetry and $3 D$ Imaging. 3rd ed., Walter de Gruyter, Berlin, 822 p, in press.

Nila, Alex. Phillips, Nathan. Bomphrey, Richard. Bleischwitz, Robert. Kat, Roeland. Ganapathisubramani, Bharathram. 2016. Optical measurements of fluid-structure interactions for the description of nature-inspired wing dynamics. RAeS Applied Aerodynamics Conference, Lisbon.

Raffel, M. Willert, C. E. Scarano, F. Kähler, C.J. Werely, S. T. Kompenhans, J. 2018: Particle image velocimetry - a practical guide-third edition. Springer Nature, Cham, $688 \mathrm{p}$.

Raguse, K. 2007: Dreidimensionale photogrammetrische Auswertung asynchron aufgenommener Bildsequenzen mittels Punktverfolgungsverfahren. Fakultät für Bauingenieurwesen und Geodäsie- Dissertation, Hannover. 
Tian, W. Ozbay, A. Hu, H. 2014: An experimental study of on the effects of incoming wind conditions on wind turbine aeromechanics. $6^{\text {th }}$ international symposium on fluid machinery and fluid engineering, Wuhan.

Weichselbaum, N.A. Hussain, S. Abkenar, M.R. Manzari, M.T. Bardet, P. M. 2016: 3D volumetric fluid-structure interaction measurements with non-intrusive optical measurement techniques on an earthquake shake table. $18^{\text {th }}$ international Symposium on the application of laser and image techniques of fluid mechanics, 18, Lisbon.

Wester, T.T.B., Kampers, G., Gülker, G., Peinke, J., Cordes, U., Tropea, C., Hölling, M. 2018: High speed PIV measurements of an adaptive camber airfoil under highly gusty inflow conditions. Journal of Physics: Conference Series, 1037, 072007

Whale, J. and Anderson, C. G. 1993: The application of particle image velocimetry to wind turbine wakes. Proceeding of SPIE The international society of optical engineering.

Winstroth, J. Schoen, L. Ernst, B. Seume, J. R. 2014: Wind turbine rotor blade monitoring using digital image correlation: a comparsion to aerolastic simulations of a multi-megawatt wind turbine. Journal of Physics: Conference Series 524 (2014) 012064 . 